IRSTI 29.27 .07

\title{
On distribution function of free and bound electrons in equilibrium Coulomb system
}

\author{
S.A. Maiorov ${ }^{1,2, *}$ and A.L. Khomkin ${ }^{1,2}$

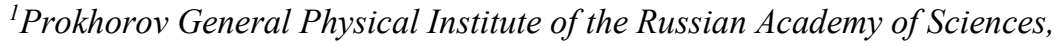 \\ 38, Vavilov Str., 119991, Moscow, Russia. \\ ${ }^{2}$ Institute for High Temperatures, Russian Academy of Sciences, \\ 13, Izhorskaya Str. Bd.2, 125412, Moscow, Russia, \\ e-mail:mayorov_sa@mail.ru
}

\begin{abstract}
In classical thermodynamics, the velocity distribution function of particles is always Maxwell distribution for any density. This is due to the fact that the dependences on the pulses and coordinates in the expression for the total energy are separated. Integration over coordinates leads to the appearance of a configuration integral, and the remaining part is divided into the product of Maxwell distribution functions. In the case of formation of bound states (molecules) in an atomic gas, the full phase space of the relative motion of two particles is divided into two parts. The first corresponds to negative energies of relative motion (molecular component), and the second to positive (free atoms). The velocity distribution function remains Maxwellian, if we ignore the fact of separation of the phase space. It can be assumed that for free atoms the velocity (kinetic energies) distribution may be different from Maxwell. For plasmas, the assumption of the non-Maxwellian velocity distribution function of free electrons was made. The influence of the non-Maxwell electron distribution function on the recombination coefficient is estimated.
\end{abstract}

Key words: distribution function, electrons, Coulomb system, recombination coefficient.

PACS numbers: 52.65. $-\mathrm{y}, 52.40 . \mathrm{Mj}$.

\section{Introduction}

The velocity distribution function of particles is always Maxwellian for any density in classical thermodynamics [1]. This is because the dependences on the pulses and coordinates in the expression for the total energy are separated. Integration over coordinates leads to the appearance of a configuration integral, and the remaining part is divided into the product of Maxwell distribution functions. Hill [2] drew attention to the fact that in the case of formation of bound states (molecules) in an atomic gas, the full phase space of the relative motion of two particles is divided into two parts. The first corresponds to negative energies of relative motion (molecular component), and the second to positive (free atoms). The velocity distribution function remains Maxwellian, if we ignore the fact of separation of the phase space. It can be assumed that for free atoms the velocity (kinetic energies) distribution may be different from Maxwell. For atomic plasmas, the assumption of the nonMaxwellian velocity distribution function of free electrons was first made in [3].

Following [3], we illustrate the above with the example of an atomic plasma, where the formation of bound states (atoms) occurs and there are free particles (electrons and ions). Considering the ions as massive particles, we write the Boltzmann distribution function for momenta $p$ and coordinates $r$, considering the interaction potential to be Coulomb:

$$
V(r)=-e^{2} / r
$$

$$
F(p, r)=\frac{1}{\left(2 \pi \hbar^{2}\right)^{3}} \int d \mathbf{p} d \mathbf{r} \exp \left(-\beta\left(\frac{p^{2}}{2 m}-\frac{e^{2}}{r}\right)\right)(1)
$$

where $\hbar$ is the Planck constant, $\beta$ is the inverse temperature, $m$ is the mass, $e$ is electron charge. Let us determine the phase space $\Omega$ for free 
electrons in a Wigner-Seitz cell of radius $a$ in order not to burden yourself considering the effect of Debye screening.

$$
\begin{gathered}
\mathbf{\Omega}=\left\{r<a ; \frac{p^{2}}{2 m}-\frac{e^{2}}{r}>-\frac{e^{2}}{a}\right\} \\
\frac{4 \pi a^{3}}{3} n_{i}=1
\end{gathered}
$$

We introduce dimensionless values and variables: $\gamma=\beta e^{2} / a, x=\beta p^{2} / 2 m, y=\beta e^{2} / r$.

Integrating over the coordinates in (1), for the distribution function of the kinetic energy we obtain:

$$
f(x, \gamma)=A(\gamma) \sqrt{x} \exp (-x) H(x, \gamma),
$$

where

$$
H(x, \gamma)=\int_{\gamma}^{x+\lambda} \frac{\exp (y)}{y^{4}} d y .
$$

In (3) $A(\gamma)$ normalization constant determined from the condition

$$
\int_{0}^{\infty} f(x, \gamma) d x=1 .
$$

Maxwell distribution in our variables is

$$
f_{M}(x)=\frac{2}{\sqrt{\pi}} \sqrt{x} \exp (-x)
$$

Figure 1 shows the distribution functions calculated by the formulas (3) and (6) for $\gamma=1$.

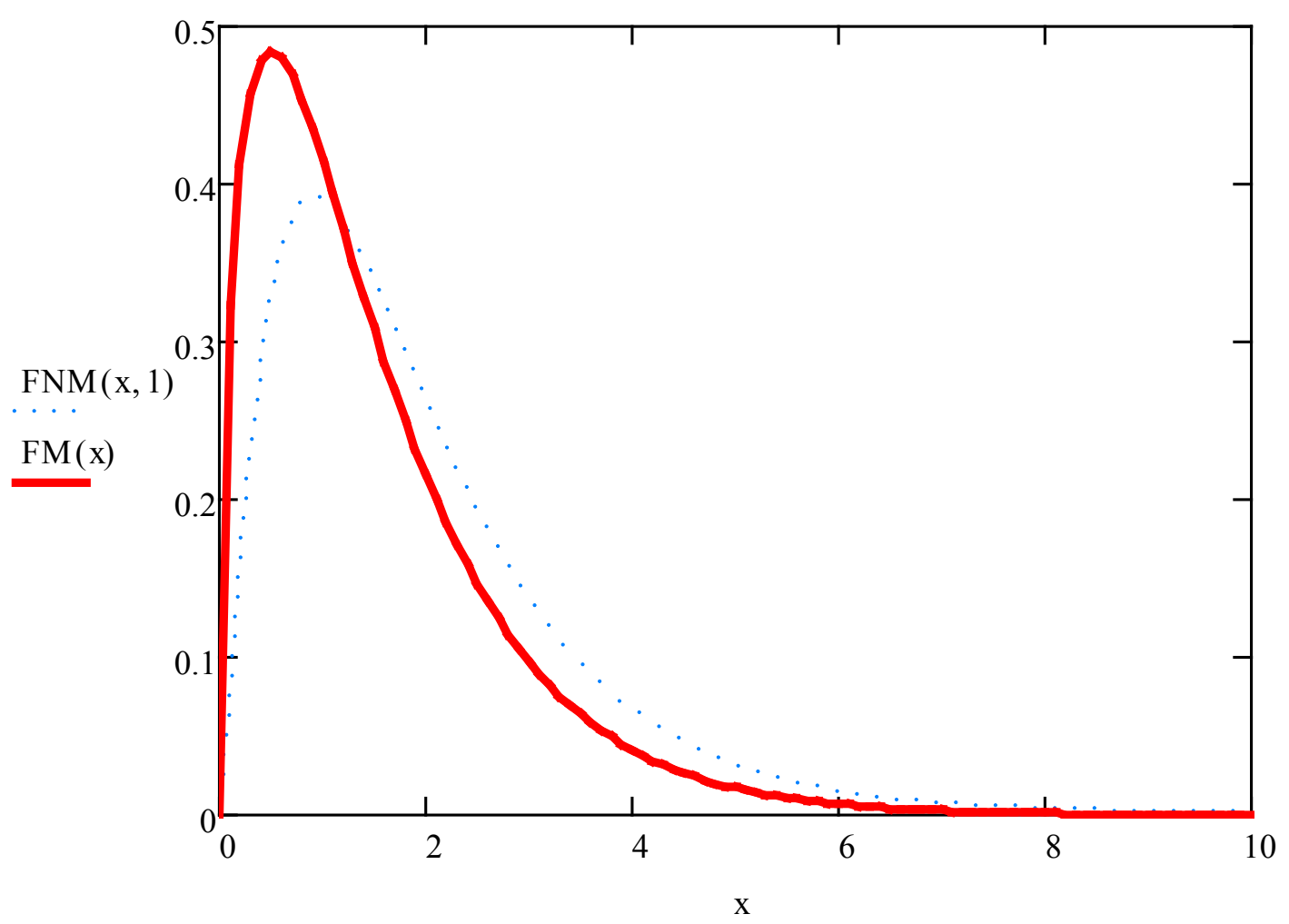

Figure 1 - The distribution function of edekronov on speeds: the solid line - Maxwell (6); dashed - nonmaxwell (3). 
It can be seen that the non-Maxwell effect manifests itself quite noticeably, especially at high kinetic energies. When confirming this effect, we can expect a noticeable modification of various kinetic coefficients, in the calculation of which it is necessary to perform velocity averaging.

The papers [4-20] is devoted to the study of the non-Maxwell configuration effect, in which the molecular dynamics method considered the relaxation of the Coulomb system, which allows the formation of bound states, to equilibrium. A similar, but pure quantum effect was considered in (see [5] and references therein), where the non-Maxwell effect was discovered by quantum statistics methods due to quantum broadening. The influence of the deviation from the Maxwell distribution on the speed of various kinetic processes was also considered. In this paper, a direct measurement of the distribution function of free electrons by kinetic energies was performed using the numerical simulation method [6] of a model Coulomb system, provided that about half of the electrons are in bound states. The non-Maxwell effect is shown explicitly.

In the present work based on the application of the molecular dynamics method:

the relaxation of the distribution function of free electrons in the course of transition to an equilibrium state is investigated;

b) equilibrium configurations of particles are calculated and the correlation function of the model Coulomb system is determined;

using statistical averaging, its thermodynamic functions are calculated.

for the first time at the microscopic level, the dynamics of the formation of bound complexes consisting of a different number of initial particles can be traced, which makes it possible to model clustering processes in the system.

\section{Calculation of parameters of the charge- symmetric model system}

Consider a model Coulomb system consisting of particles of the same mass. Choose the model potential of interaction between particles in the form:

$$
V(r)=\left\{\begin{array}{l}
e^{2} / \sigma\left[(\sigma / r)^{12}-(\sigma / r)\right], \text { in }+- \text { interaction, } \\
e^{2} / r, \text { in }++,-- \text { interaction. }
\end{array}\right.
$$

Its asymptotic behavior for opposite charges at small distances $r$ has the character of repulsion, and with $\mathrm{r} \rightarrow \infty$ it is attractive and coincides with the Coulomb potential $1 / \mathrm{r}$. For intermediate values of $r$, the potential has a well and allows the formation of classical bound states. The choice of the model potential in the form of (7) is due to the need to eliminate the Coulomb potential feature at $r \rightarrow 0$, which makes it impossible to integrate the equations of motion of particles. At such a potential, bound states are formed in the system. The thickness of the soft wall $\sigma$ can always be chosen equal to the diameter of the ion core. In the present work, the choice of potential parameters: $\sigma$ and $\varepsilon=\mathrm{e} 2 / \sigma-$ the depth of the potential well was carried out in such a way as to realize the required values of the nonideality parameter $\Gamma$ and the degree of ionization $\alpha(\sigma=2 \AA)$. The latter was estimated by the classical Saha formula with the potential 12-1 (7).

The temporal evolution of the system was modeled using the multi-particle dynamics method (DMP) $[4,6,16-20]$. The trajectories of $\mathrm{N}$ positive and $\mathrm{N}$ negatively charged ions were determined by numerical solution of Newton's

$$
\begin{gathered}
\ddot{r}_{k}=F_{k} / m_{k}, F_{k}=\sum_{l \neq k}^{2 n} f_{k l}, k=1,2, \ldots, 2 N, \\
f_{k l}=\left\{\begin{array}{c}
\frac{e^{2}\left(r_{k}-r_{l}\right)}{\left|r_{k}-r_{l}\right|^{3}} \\
\frac{e^{2}\left(r_{k}-r_{l}\right)}{\left|r_{k}-r_{l}\right|^{2} \sigma}\left[\left(\frac{\sigma}{r}\right)^{12}-\left(\frac{\sigma}{r}\right)\right],
\end{array}\right.
\end{gathered}
$$

where rk is the radius vector of the particle number $\mathrm{k}$; $\mathrm{mk}$ is it's mass (the mass of protons was taken); e is electron charge.

At the initial time $\mathrm{t}=0$ the coordinates and velocities of the particles were set using a random number generator. The coordinates are in accordance with the uniform distribution, and the speed in accordance with the Maxwell distribution for the initial temperature T0. Maxwell distribution was defined as follows:

$$
\begin{aligned}
& V_{x}=\sqrt{-2 \overline{V^{2}} \ln \xi_{1}} * \cos \left(2 \pi \xi_{3}\right) \\
& V_{y}=\sqrt{-\overline{2 V^{2}} \ln \xi_{1}} * \sin \left(2 \pi \xi_{3}\right) \\
& V_{z}=\sqrt{-2 \overline{V^{2}} \ln \xi_{2}} * \cos \left(2 \pi \xi_{4}\right)
\end{aligned}
$$




$$
\begin{gathered}
\xi \in(0,1) \\
\bar{V}^{2}=k T / m
\end{gathered}
$$

The particles were in a cube with thermostatic walls. The length of the cube edge was chosen such as to ensure the desired density of particles. Particles reaching the walls returned to the cube with an equilibrium distribution of kinetic energy corresponding to the wall temperature Tst.

In the calculations, the algorithm [4] was used to reduce computer time. Let at the time t0 be known all coordinates $\mathrm{rk}(\mathrm{t} 0)$ and velocities $\mathrm{vk}(\mathrm{t} 0)$ of particles. The procedure for determining the quantities $\operatorname{rk}(\mathrm{t} 0+\Delta \mathrm{t})$ and $\mathrm{vk}(\mathrm{t} 0+\Delta \mathrm{t})$, where $\Delta \mathrm{t}-\mathrm{a}$ step in time, is as follows.

a). The coordinate values $\operatorname{rok}(\mathrm{t} 0+\Delta \mathrm{t} / 2)$, corresponding to the rectilinear motion of the particles, are calculated.

b). The values of the forces acting on the particles are calculated:

$$
F_{k}^{c}\left(t_{0}+\Delta t / 2\right)=\sum_{l \neq k}^{2 N} f_{k l}\left(r_{k}^{0}-r_{l}^{0}\right)
$$

c). Each particle is determined by the two nearest neighbors of the particles of each sign and the distance to them.

d). The force acting on a particle is calculated as the sum of two forces

$$
F_{k}=F_{k}^{c}+F_{k}^{\tau}
$$

where $F_{k}^{\tau}$ due to the interaction with the nearest neighbors of a given particle, $F_{k}^{c}$-interaction with all other particles.

e). Newton's equations are integrated according to the fourth-order Runge-Kut scheme with a step

$$
\tau=\Delta \mathrm{t} / \mathrm{N} \tau
$$

where $\mathrm{N} \tau$ is the number of internal steps.

In the course of integration, only interaction with the nearest particles is considered temporary.

$$
\begin{aligned}
& F_{k}(t)=F_{k}^{c}\left(t_{0}+\Delta t / 2\right)+ \\
& +F_{k}^{\tau}(t), t_{0}<t<t_{0}+\Delta t,
\end{aligned}
$$

After determining the values $\operatorname{rk}(\mathrm{t} 0+\Delta \mathrm{t})$ and $\operatorname{vk}(\mathrm{t} 0+\Delta \mathrm{t})$ the condition of the particles leaving the cube boundary is checked. Particles that go beyond the cube bound are returned back to the cube with an equilibrium distribution of kinetic energy corresponding to the wall temperature Tst.

The following values were used in the calculations:

$$
\Delta \mathrm{t}=0.05 \tau \mathrm{ei}, \mathrm{N} \tau=10,
$$

where $\tau$ ei is the mean time interparticle distance.

\section{The simulation results and discussion}

At the first stage, the relaxation of a system of charges of equal mass to an equilibrium state was studied, which was fixed by the temperature and ionization degree to equilibrium values.

Figure 2 shows the dependence of the number of bound states on time. A system of 400 negative and the same number of positive ions is considered. External parameters: density $\mathrm{nk}=1020 \mathrm{~cm}-3$ and temperature $\mathrm{T}=1200 \mathrm{~K}$. The degree of ionization, calculated according to the classical Saha formula, was about 0.5 . The exit of the system to ionization equilibrium is clearly visible. The wave-like nature of relaxation can be associated with the reflection of a diffusion flow of bound particles from the bottom of a potential well. Such a relaxation pattern is observed in all the considered modes. The relaxation time increases with decreasing of density. For $\mathrm{nk}=1019 \mathrm{~cm}-3$ time to equilibrium is about $2500 \tau$ ei. 


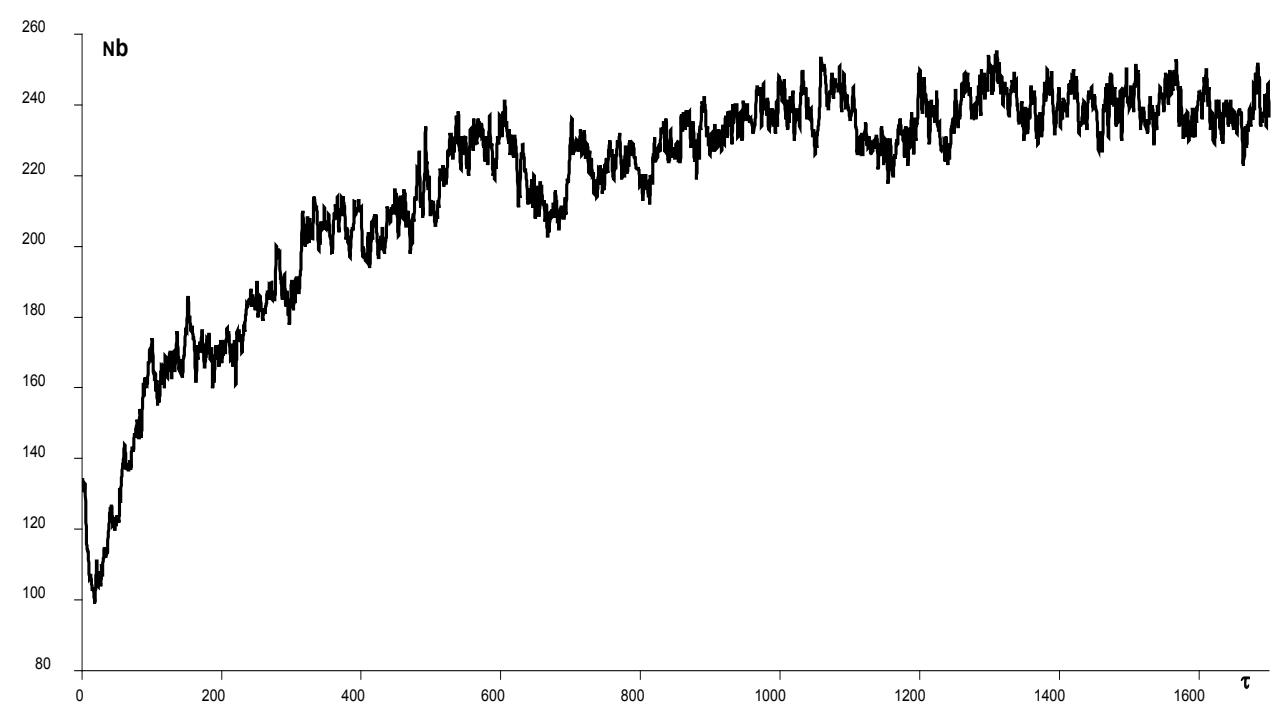

Figure 2 - The dependence of the number of bound states on time (ne $=1020 \mathrm{~cm}-3$ and $\mathrm{T}=1200 \mathrm{~K}$ )

Figure 3 shows the particle temperature relaxation (two thirds of the average kinetic energy) for the same mode as in Figure 1. The previous stage can be considered as preparation for the process of measuring equilibrium characteristics. In the equilibrium region, the particle distribution function was measured by kinetic energy and distances to the nearest ion. Averaging was performed over several dozens of equilibrium configurations. Measurements were carried out both for free particles and for particles bound into atoms.

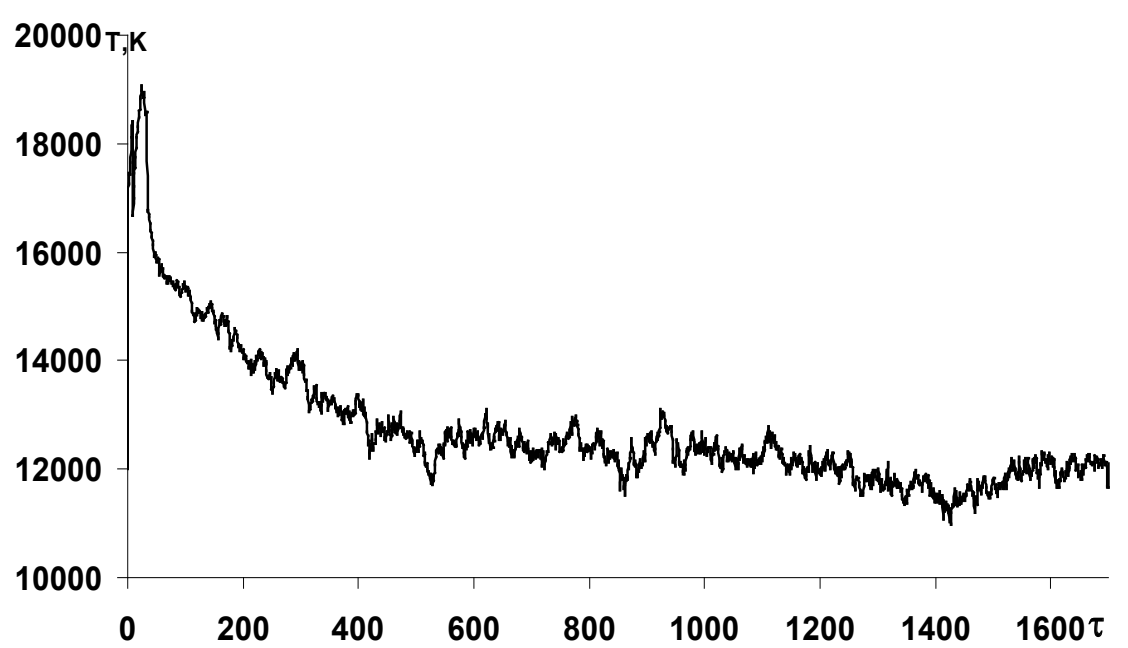

Figure 3 - Particle temperature relaxation (ne $=1020 \mathrm{~cm}-3$ and temperature $T=1200 \mathrm{~K}$ ).

Figures 4 a), b), c) show the measured distribution functions of free negatively charged ions by kinetic energies $(\varepsilon-$ is the dimensionless kinetic energy related to temperature) for the three regimes in density and temperature. 


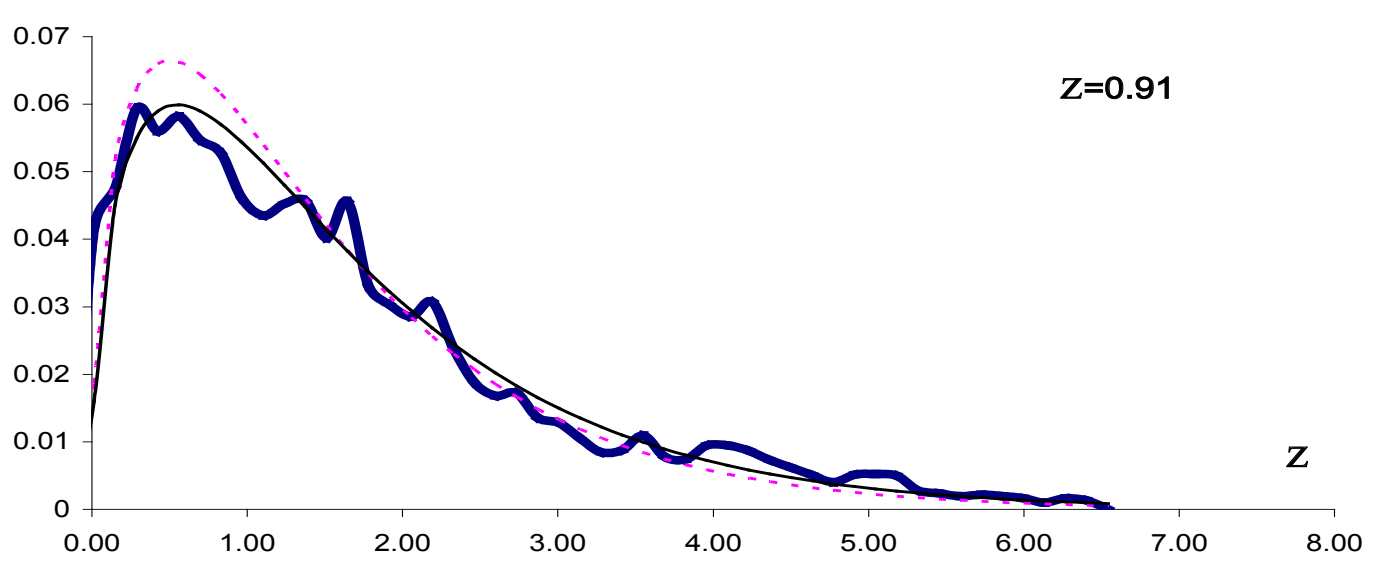

a)

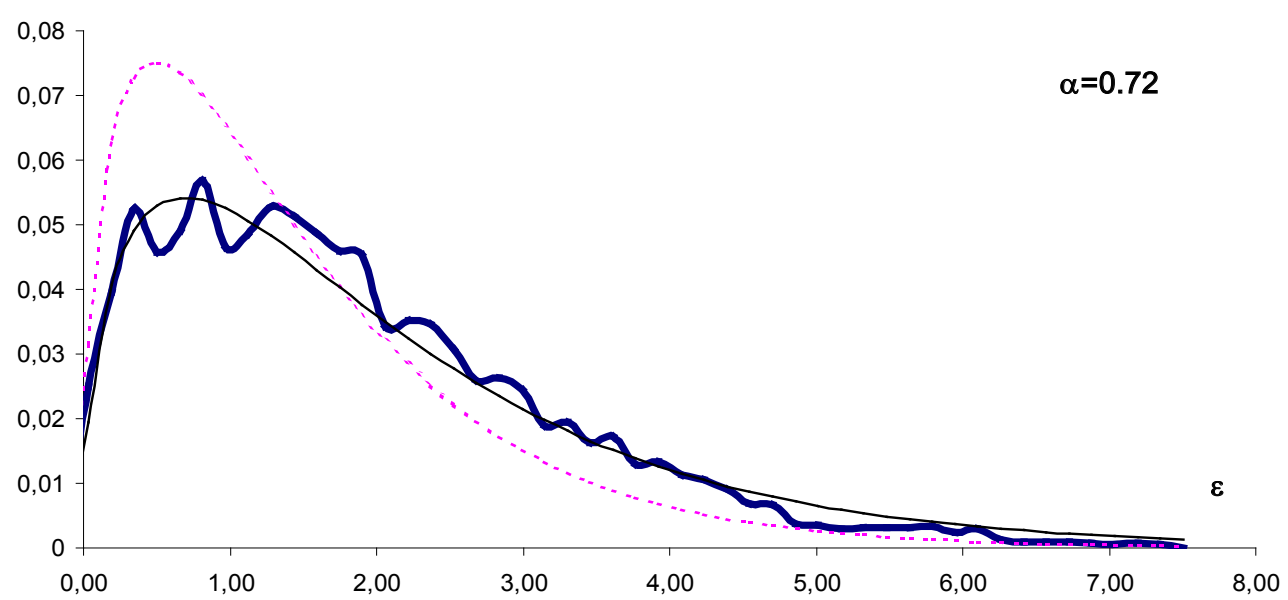

b)

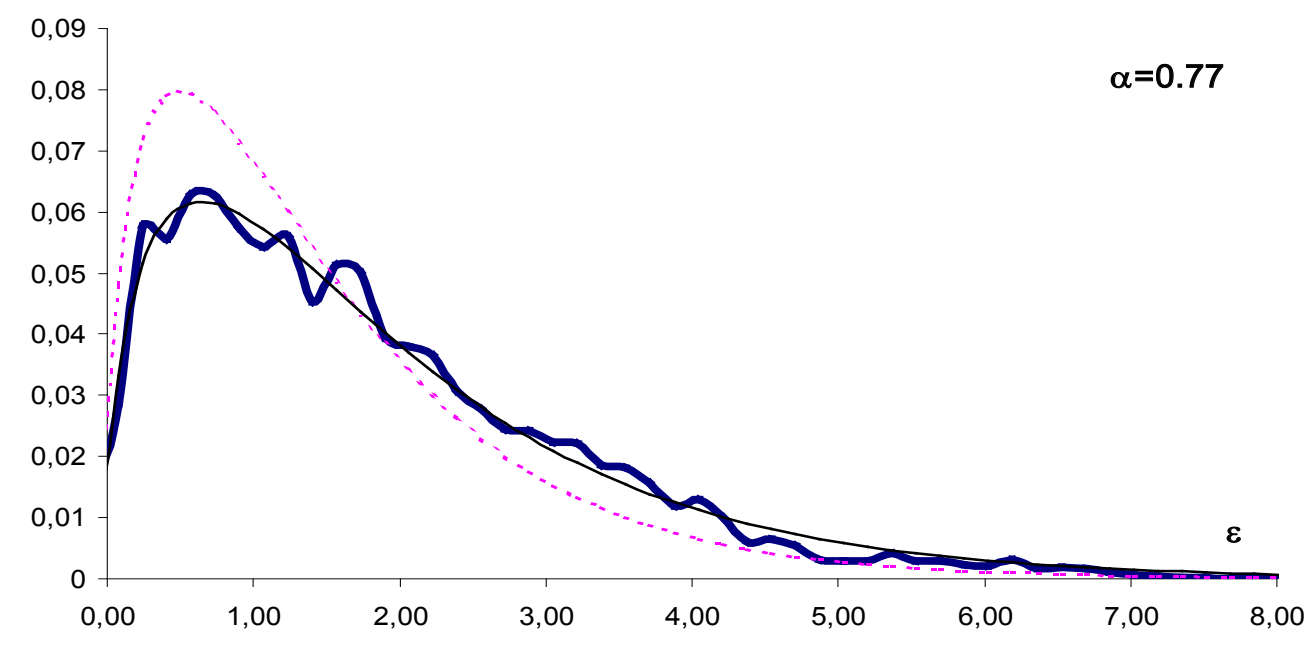

c)

Figure 4 - The distribution function of free negatively charged ions by kinetic energy:

a). ne $=1019 \mathrm{~cm}-3$ and $\mathrm{T}=8000 \mathrm{~K}, \mathrm{~b}) . \mathrm{ne}=1020 \mathrm{~cm}-3$ and $\mathrm{T}=12000 \mathrm{~K}, \mathrm{c}) . \mathrm{ne}=1021 \mathrm{~cm}-3$ and $\mathrm{T}=18000 \mathrm{~K}$. 
Solid smooth lines correspond to the function of the form:

$$
f(\varepsilon)=\frac{2}{\sqrt{\pi}} \sqrt{\varepsilon} \alpha^{\frac{3}{2}} \exp (-\alpha \varepsilon) .
$$

with the values of the parameter $\alpha$, indicated in the figures. The deviation of the equilibrium distribution function of free particles from the Maxwell distribution is quite clearly seen. With increasing nonideality, these deviations increase. At low energies, there is a shortage, and at high energies, an excess of free ions. Qualitatively, this behavior corresponds to the results of [3]. It is noticed that in the system with the formation of bound states there appear molecules consisting of more than two particles. The following connectivity criterion was chosen: a pair of particles is connected if the total energy of their relative motion is less than the interaction energy at an average distance. All bonds in the system were considered and the molecular composition was analyzed. After that, averaging was performed over several dozens of equilibrium configurations.

Figure 5 shows how many molecules of each type are represented in the system; Nat is the number of particles in a molecule. It can be seen from the figure that as the density increases, the specific gravity of the pairs decreases, and more and more heavy molecules appear. Numerical calculation shows that the lifetime of molecules consisting of more than four particles does not exceed 20 characteristic times, whereas the average lifetime of pairs is about 100 characteristic times.

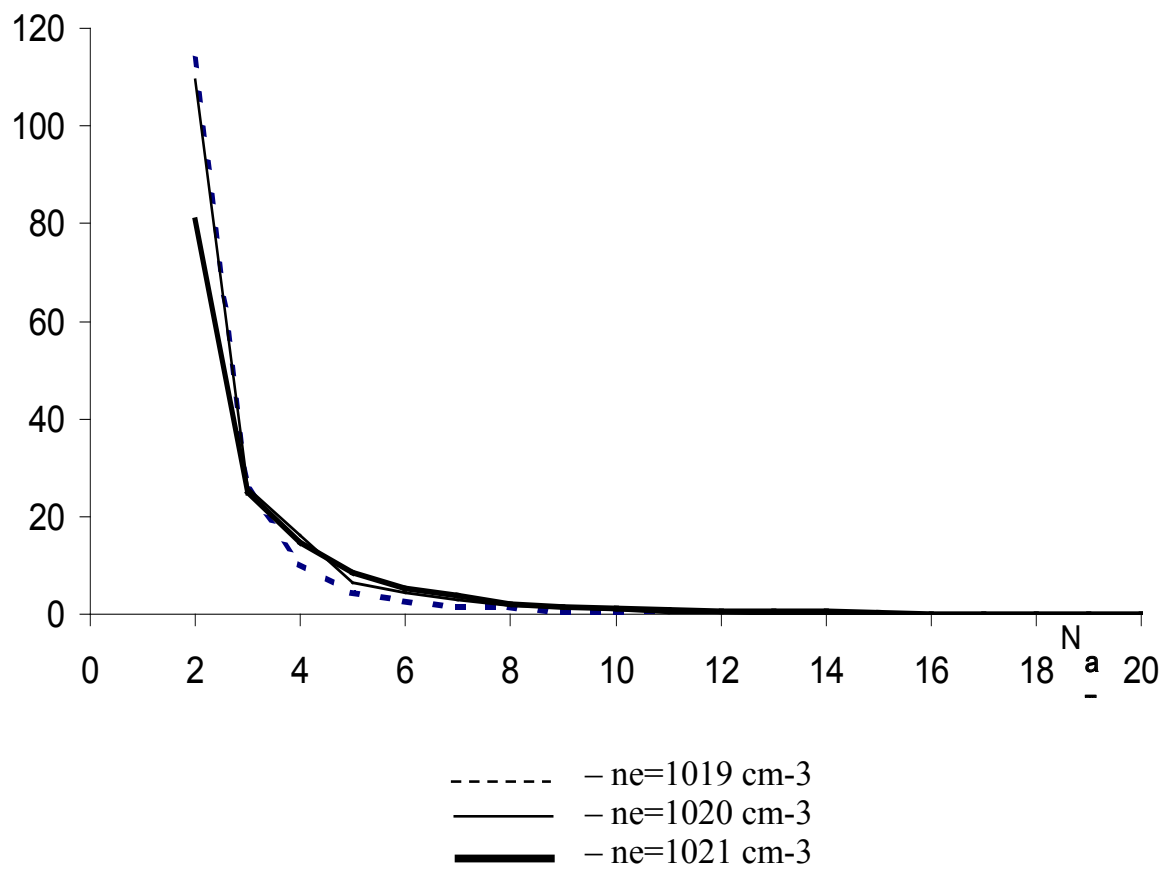

Figure 5 - The average number of polyatomic complexes in the system

Figure 6 shows the charge distribution of molecules. It can be seen that the highest number is represented by singly charged molecules.

We also measured the average energy of the interaction of a free electron with the system. It was taken into account that some heavy molecules were charge carriers, which had an additional effect on the motion of free particles. The obtained data are presented in Figure 7. Solid thick line - the energy of the interaction of particles at a medium distance, the dashed line and the double dashed line are the Debye theory in the large and small canonical ensemble, respectively, the data of molecular dynamics calculations are shown by dots. It can be seen that the measured average interaction energy of a free electron with the entire system turns out to be close to the interaction energy of particles at an average distance, but slightly exceeds the latter. 


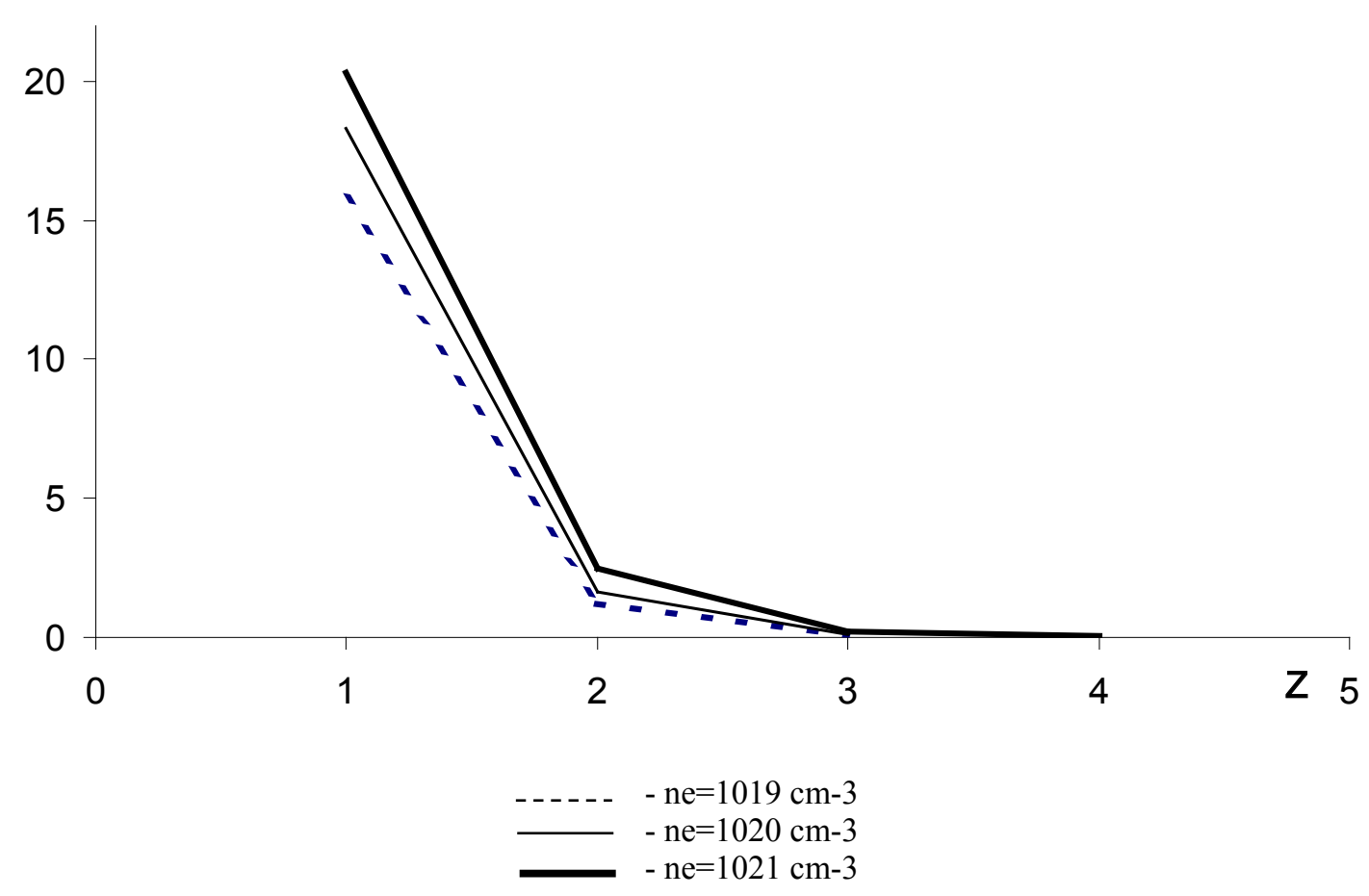

Figure 6 - The distribution of polyatomic complexes on charges

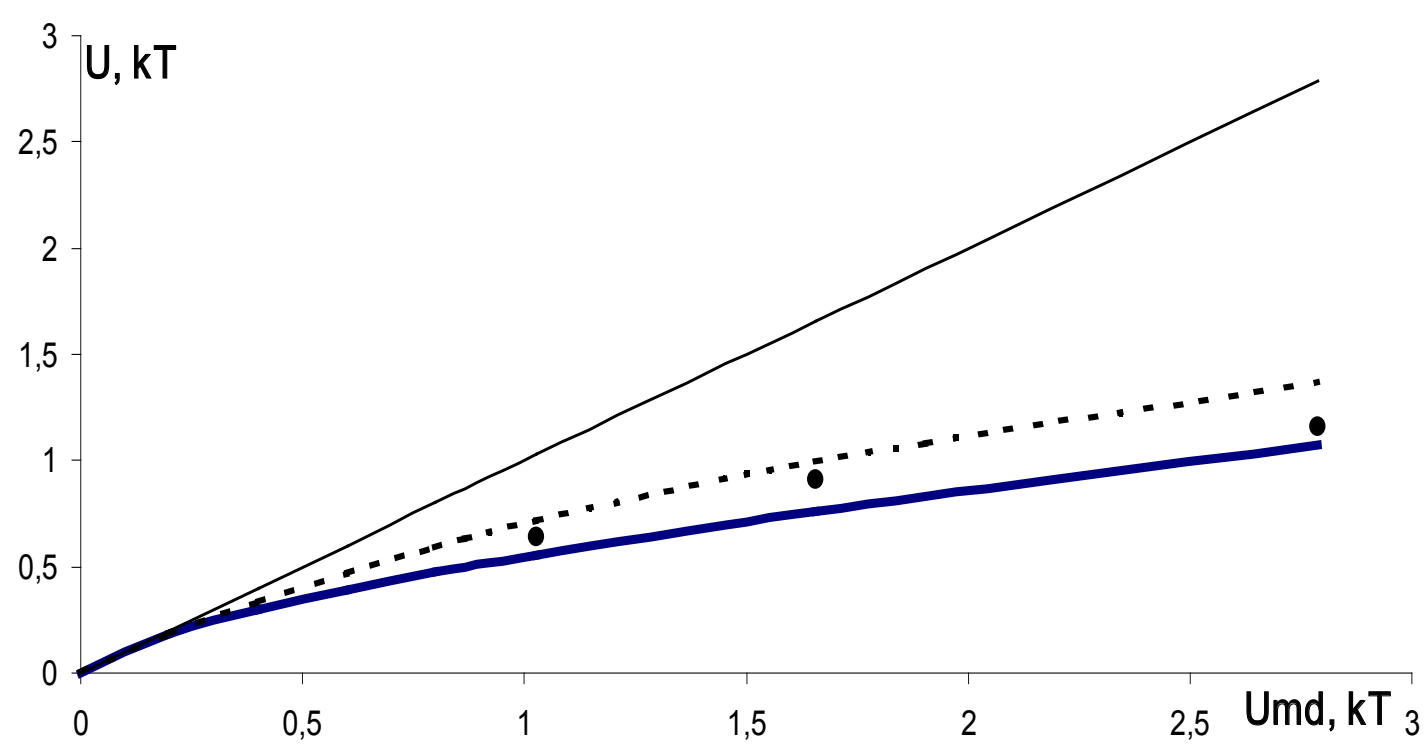

- interaction energy of particles at an average interparticle distance,

- the Debye approximation in the large and small canonical ensemble, respectively, $-^{-\cdots--}$ - the results of a numerical experiment

Figure 7 - The average energy of the interaction of a free electron with free particles 
The relaxation of model Coulomb systems is studied by the molecular dynamics method in papers [7-9] and the recombination coefficient in a nonideal plasma is calculated. The flux in the energy space is calculated in [7], and in [8] the increase of the number of bound states is analyzed, which are determined by the number of revolutions of the electron around the ion. In the present work, we evaluated the role of non-Maxwell in the recombination coefficient. In accordance with Thomson's theory, the recombination frequency is proportional to the velocity of the incident electron, the scattering cross section, and the cube of the Landau length, which leads to the dependence $T^{-9 / 2}$

The correction factor was calculated by the formula

$$
K(\gamma)=\frac{\left\langle\left(\frac{p^{2}}{2 m}\right)^{9 / 2}\right\rangle_{\operatorname{Max}}}{\left\langle\left(\frac{p^{2}}{2 m}\right)^{9 / 2}\right\rangle_{\text {Non Max }}}
$$

where in the numerator averaging was performed with the Maxwell distribution function (6), and in the denominator with (3).

Using the expression for the Thomson recombination coefficient $\alpha$

$$
\alpha=2.07 \frac{e^{10}}{\sqrt{m} T^{9 / 2}}
$$

we get $\alpha_{N M}=\alpha K(\gamma)$. Figure 8 presents theoretical dependencies $\alpha$ and $\alpha_{N M}$ from $\gamma$ in coordinates $\alpha n_{e} / \omega_{p}, \gamma$, as well as numerical simulation data $[7,8]$ and [9].

\section{Conclusions}

This paper investigates the relaxation of a charge-symmetric system of Coulomb particles to an equilibrium state. It is shown that in the system with the selected interaction potential (7) bound states arise. The temperature relaxation process coincides in time with the establishment of ionization equilibrium between bound and free states. A direct "measurement" of the distribution function of free particles by the kinetic energy in the equilibrium state was carried out. Its deviation from Maxwellian, due to a decrease in the configurational space of free particles, was recorded. The free charge subsystem turned out to be "hotter" than all the charges in general. The kinetic energy distribution of the entire set of charges (bound and free) remains Maxwellian.

The influence of the non-Maxwell of the electron distribution function on the recombination coefficient is estimated.

The relaxation of the radial distribution function of all particles is investigated in the approximation of the nearest neighbor. At equilibrium, the radial distribution function measured in this way coincides with the theoretical one within the error of averaging.

The measured average interaction energy of a free electron with the entire system is close to the interaction energy of particles at an average distance, which indicates the saturation of the Coulomb nonideality in the system of free charges. In the subsystem of bound particles with increasing density, along with paired particles, polyatomic complexes are also formed, i.e. there is a clustering process. 


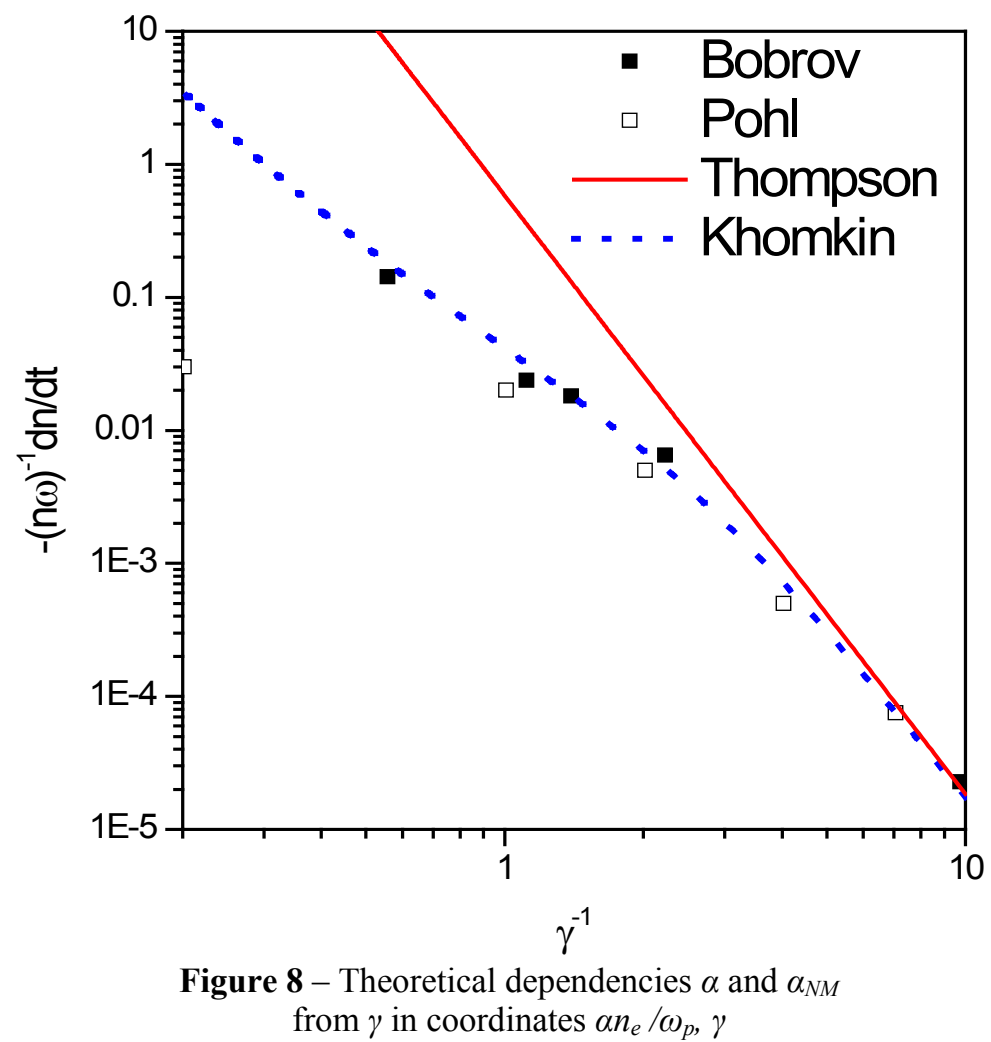

Thus, the main effect of nonideality in the formation of composite complexes, while the system with increasing density is associated with the interaction energy of free charges grows slightly.

\section{References}

1. L.D. Landau, E.M. Lifshitz. Course of Theoretical Physics. Volume 5: Statistical Physics, Part 1, Oxford: Butterworth-Heinemann, 1975, $584 \mathrm{p}$.

2. T. Hill. Statistical mechanics. Principles and selected applications. New York a. o., McHaw-Hill, 1956, 520 p.

3. T.P. Wright, O. H. Theimer. Non-Maxwellian equilibrium distribution for free electrons in a plasma // Phys. Fluids. -1970. - Vol.13. - No.4. -Pp. 895-901.

4. S. Novikov, A.L. Khomkin. Investigation of relaxation and equilibrium properties of a non-ideal Coulomb system using the molecular dynamics method // Proceedings of the Conference FNTP-98, Petrozavodsk, 1998, part 1. 1998. -Vol. 2. -Pp.476-479.

5. A.V. Eletsky, A.N. Starostin, M.D. Taran. Quantum corrections to the equilibrium rate constants of inelastic processes // Phys.-Usp. - 2004. -Vol. 175. -No.3. -Pp. 299-313.

6. S.A. Maiorov, A.N. Tkachev, S.I. Yakovlenko. Studies of the fundamental properties of Coulomb plasma by the method of the dynamics of many particles // Sov. Phys. J. -1991. -Vol. 34. -No.11. -P. 951.

7. A.A. Bobrov, S.Ya. Bronin, B.B. Zelener, B.V. Zelener, E.A. Manykin, D.R. Khikhlukha. Collision recombination coefficient in ultracold plasma. Molecular dynamics calculation // JETP. - 2011. - Vol. 139. -Pp. 605.

8. A. V. Lankin, G. E. Norman. Collisional recombination in strongly coupled plasma // J. Phys. A. - 2009. -Vol. 42. -P. 214032.

9. G. Bannasch, T. Pohl. Rydberg atom formation in strongly correlated ultracold plasmas // Phys. Rev.A. - 2011. Vol. 84. - P. 052710.

10. D.N. Zubarev. Nonequilibrium statistical thermodynamics, Berlin: Springer Verlag, 1974, 416 p.

11. P. Landsberg. Problems in thermodynamics and statistical physics, Ed., London: Pion, 1989, 640 p. 
12. A. Isihara. Statistical Physics, New York: Academic, 1971, 472 p.

13. Yu.L. Klimontovich. Statistical Physics, Boca Raton, Florida, United States: CRC Press, 1986, 608 p.

14. Ya.P. Terletskii. Statistical Physics, Amsterdam, The Netherlands: North Holland, 1971, 280 p.

15. J. Mayer, M. Goeppert-Mayer. Statistical Mechanics, New York: Wiley, 1977, 544 p.

16. S.A. Maiorov, A.N. Tkachev, S.I. Yakovlenko. Unexpected properties of classical Coulomb plasma, discovered on the basis of first principles modeling // Mat. Model. - 1992. -Vol. 4. - No.7. - P. 3. (in Russian)

17. S.A. Maiorov, A.N. Tkachev, S.I. Yakovlenko. Recombination on of a Coulomb plasma and nonbinary interaction effects // Russ. Phys. J. - 1993. -Vol.36., No.1. - P. 55.

18. S.A. Maiorov, S.I. Yakovlenko. S.I. Development of a method for modeling the dynamics of many coulomb particles // Russ. Phys. J. - 1994. - Vol. 37., No. 11. - P.1048.

19. S.A. Maiorov, A.N. Tkachev, S.I. Yakovlenko. Metastable supercooled plasma // Phys. - Usp. - 1994. - Vol. 37., No. 3. - P. 279.

20. S.A. Maiorov. Effect of ion collisions on the parameters of dust-plasma structures // Plasma Phys. Rep. - 2006. - Vol. 32., No. 9. - P. 737. 